\title{
Enhancing aperture efficiency of reflectarray by accurately evaluating mutual coupling of reflectarray elements
}

\author{
Keisuke Konno ${ }^{\text {a) }}$ and Qiang Chen \\ Department of Communications Engineering, \\ Graduate School of Engineering, Tohoku University, \\ 6-6-05 Aramaki Aza Aoba, Aoba-ku, Sendai-shi, Miyagi 980-8579, Japan \\ a)konno@ecei.tohoku.ac.jp
}

\begin{abstract}
In this letter, aperture efficiency of reflectarrays is enhanced using an efficient full-wave method. The full-wave method deals with the effect of real mutual coupling between all reflectarray elements exactly during its design process. Resultant long CPU time is greatly reduced by developing an efficient algorithm which is optimized for the vector supercomputer. Numerical simulation shows that the aperture efficiency of the reflectarrays can be enhanced using the proposed method. The proposed method is computationally efficient and applicable for various design targets.
\end{abstract}

Keywords: reflectarray, vector supercomputer

Classification: Antennas and Propagation

\section{References}

[1] D. G. Berry, R. G. Malech, and W. A. Kennedy, "The reflectarray antenna," IEEE Trans. Antennas Propag., vol. 11, no. 6, pp. 645-651, Nov. 1963. DOI:10.1109/TAP.1963.1138112

[2] J. Huang, "Analysis of a microstrip reflectarray antenna for microspacecraft applications," TDA Progress Report 42-120, pp. 153-173, Feb. 1995.

[3] J. Huang and J. A. Encinar, Refrectarray Antennas, John Wiley and Sons, 2008.

[4] C. Wan and J. A. Encinar, "Efficient computation of generalized scattering matrix for analyzing multilayered periodic structures," IEEE Trans. Antennas Propag., vol. 43, no. 11, pp. 1233-1242, Nov. 1995. DOI:10.1109/8.475095

[5] L. Li, Q. Chen, Q. Yuan, K. Sawaya, T. Maruyama, T. Furuno, and S. Uebayashi, "Novel broadband planar reflectarray with parasitic dipoles for wireless communication applications," IEEE Antennas Wireless Propag. Lett., vol. 8, pp. 881-885, 2009. DOI:10.1109/LAWP.2009.2028298

[6] L. Li, Q. Chen, Q. Yuan, K. Sawaya, T. Maruyama, T. Furuno, and S. Uebayashi, "Frequency selective reflectarray using crossed-dipole elements with square loops for wireless communication applications," IEEE Trans. Antennas Propag., vol. 59, no. 1, pp. 89-99, Jan. 2011. DOI:10.1109/TAP. 2010.2090455

[7] F. Venneri, G. Angiulli, and G. Di Massa, "Design of microstrip reflectarray 
using data from isolated patch analysis," Microw. Opt. Technol. Lett., vol. 34, no. 6, pp. 411-414, Sept. 2002. DOI:10.1002/mop.10479

[8] M.-A. Milon, D. Cadoret, R. Gillard, and H. Legay, "'Surrounded-element' approach for the simulation of reflectarray radiating cells," IET Microw. Antennas Propag., vol. 1, no. 2, pp. 289-293, April 2007. DOI:10.1049/ietmap:20050291

[9] C. Yann, R. Loison, R. Gillard, M. Lebeyrie, and J.-P. Martinaud, “A new approach combining surrounded-element and compression methods for analyzing reconfigurable reflectarray antennas," IEEE Trans. Antennas Propag., vol. 60, no. 7, pp. 3215-3221, July 2012. DOI:10.1109/TAP.2012. 2196938

[10] C.A. Balanis, Antenna Theory Analysis and Design, 3rd ed., pp. 461-467.

[11] K. Konno, Q. Chen, K. Sawaya, S. Kameda, and N. Suematsu, "Novel design method for reflectarray by induced electromotive force method," Proc. IEEE AP-S Int. Symp., 429.3, pp. 1342-1343, July 2013.

\section{Introduction}

A reflectarray has received much attention as a compact and light weight reflector antenna $[1,2,3]$. The reflectarray is a so-called semi-periodic structure because array itself is periodic while every element has a different dimension. In order to design the reflectarray, phase of reflection coefficient is obtained in advance as a function of dimension of reflectarray element. However, it is difficult to obtain the phase of reflection coefficient exactly because the effect of real mutual coupling is unknown before the reflectarray structure is finally designed. As a result, phase of reflection coefficient is obtained by using various techniques which deal with the effect of mutual coupling approximately.

It is very popular to obtain the phase of reflection coefficient using an unit cell analysis under periodic boundary condition $(\mathrm{PBC})[4,5,6]$. It is known that the unit cell analysis under the PBC works well when the dimension of reflectarray element varies continuously in the reflectarray. However, in practice, the dimension of the reflectarray element often varies discontinuously and so-called local periodicity cannot be assumed. In addition, a real reflectarray is a finite structure, but not an infinite structure. As an alternative approach, isolated element approach [7] and surrounded element approach $[8,9]$ have been proposed respectively. The isolated element approach neglects the effect of mutual coupling while the surrounded element approach assumes mutual coupling in a finite array. Both of these approaches deal with the reflectarray as a finite structure but the effect of real mutual coupling cannot be reflected to the phase of reflection coefficient. To the best of our knowledge, design of reflectarray which deals with the real mutual coupling between reflectarray elements has not been performed yet.

In this letter, the aperture efficiency of reflectarrays is enhanced using an efficient full-wave solver and the real mutual coupling between reflectarray elements is dealt with exactly except for the setup of the initial reflectarrays. Initial dimensions of every reflectarray element in reflectarrays are given using conventional isolated or surrounded element approach. After that, dimensions of every reflectarray element are renewed in order to enhance the gain of reflectarrays. The 
performance of the resultant reflectarray is evaluated using the full-wave solver every time dimensions of reflectarray element are renewed.

\section{Proposed design method of reflectarray}

A structure of reflectarray is shown in Fig. 1. A metal-only reflectarray which consists of a linear element is assumed here and a full-wave analysis was performed using induced electromotive forced (EMF) method $[10,11]$. In order to reduce the number of unknowns as much as possible, two types of reflectarray elements, i.e. a linear dipole element $\mathrm{w} /$ and $\mathrm{w} / \mathrm{o}$ parasitic dipole elements are mixed. In this letter, the linear dipole element $\mathrm{w} /$ parasitic dipole elements is deployed only when the linear dipole element cannot compensate the required phase shift. Dimensions of the two kinds of reflectarray elements except for their length were optimized in advance in order to have a large phase shift as much as possible using the isolated or surrounded element approach. Although a horn antenna is often used as a primary source of reflectarrays in practice, a dipole antenna was used as a primary source to simplify the numerical simulation here because this letter focuses on the real mutual coupling effect between reflectarray elements, not primary source. The effect of an infinite ground plane was included in our numerical simulation using the image theory.

Design of reflectarrays which deals with the real mutual coupling is performed as follows.

1. Phase of reflection coefficient of reflectarray elements is calculated approximately using isolated or surrounded element approach.

2. Initial length of every reflectarray element is obtained using the phase of reflection coefficient.

3. Length of $m$ th reflectarray element is incremented/decremented from $l_{m}$ to $l_{m} \pm \Delta l$ and impedance matrix of the entire reflectarray is renewed. Here, $l_{m}$ is the initial length of $m$ th reflectarray element and $\pm \Delta l$ is its increment/ decrement.

4. Gain $G\left(l_{m} \pm \Delta l\right)$ of the renewed reflectarray is obtained using full-wave solver based on a preconditioned conjugate gradient method.

5. If $G\left(l_{m}+\Delta l\right)>G\left(l_{m}\right)$ and $G\left(l_{m}+\Delta l\right)>G\left(l_{m}-\Delta l\right)$, the length of $m$ th reflectarray is incremented until $G$ reaches local maximum value. If $G\left(l_{m}-\Delta l\right)>$ $G\left(l_{m}\right)$ and $G\left(l_{m}-\Delta l\right)>G\left(l_{m}+\Delta l\right)$, the length of $m$ th reflectarray is decremented until $G$ reaches local maximum value. If $G\left(l_{m}\right)>G\left(l_{m}+\Delta l\right)$ and $G\left(l_{m}\right)>G\left(l_{m}-\Delta l\right)$, the length of the $m$ th reflectarray element is kept.

6. $3 \sim 5$ is repeated until $m$ reaches to $M$, where $M=M_{x} M_{y}$ is the number of reflectarray elements.

It should be indicated that the design method only gives the suboptimum design because no global optimization technique is used. However, the real mutual coupling is dealt with exactly and the resultant reflectarray is expected to have an enhanced performance.

In order to reduce the long CPU time of the numerical simulation, a vector supercomputer SX-ACE at Cyberscience Center, Tohoku University was used. It is well known that the vector supercomputer performs so-called vector operation 


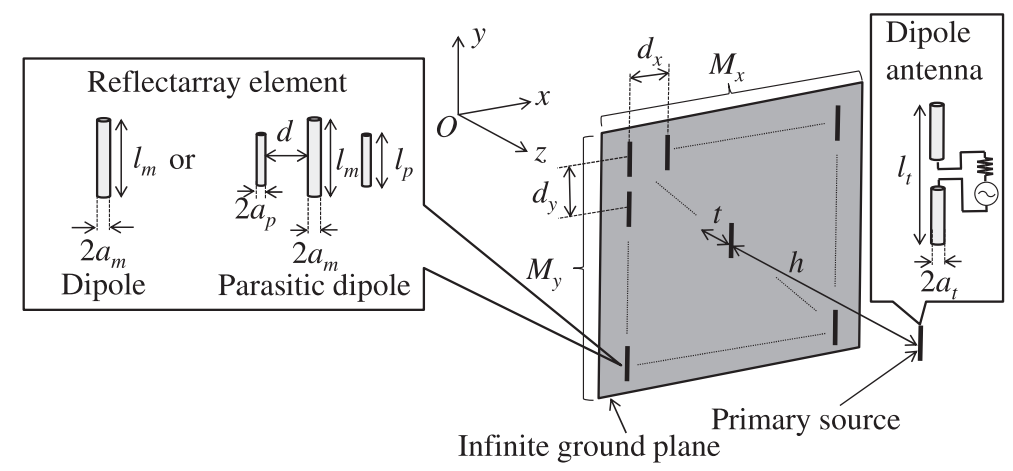

Fig. 1. A linear element reflectarray.

which deals with iterative operations simultaneously. Therefore, outstanding computing performance is expected when the vector operation is fully utilized. Our inhouse code was optimized for the SX-ACE in order to maximize vector operation ratio which shows ratio of vector operation to total operation. As a result, vector operation ratio over $99.2 \%$ was achieved by tuning the program carefully.

\section{Numerical results}

$50 \times 50$ reflectarrays were designed using the isolated and surrounded element approach combined with the proposed method. Here, phase of reflection coefficient of reflectarray elements in the center of $3 \times 3$ array of identical elements was used for surrounded element approach. Gain of the reflectarrays is shown in Figs. 2(a) 2(d). It is found that the gain of the reflectarrays designed using conventional isolated and surrounded element approach is enhanced after the proposed method is applied. Enhancement of the gain is caused by accurately evaluating mutual coupling between reflectarray elements. In other words, the proposed method can utilize the effect of real mutual coupling between reflectarray elements in order to enhance the gain. On the other hand, it is demonstrated that the gain of the reflectarrays designed using the isolated or surrounded element is quite low when their array spacing is small. This is because the effect of real mutual coupling on the gain of the reflectarrays is strong when the array spacing is small and should be dealt with exactly when the reflectarrays are designed. As shown in Fig. 2(a) and 2(c), the proposed method works well even when the array spacing of the reflectarrays is small.

In order to clarify the effect of real mutual coupling on the performance of the reflectarrays, the aperture phase distribution of the reflectarrays is shown in Figs. 2(e) 2(h). Figs. 2(e) 2(h) imply that the dimensions of a reflectarray element vary discontinuously and have no local periodicity especially when the array spacing is small. Therefore, it can be said that the performance of the reflectarrays which are designed using any of conventional approaches (i.e. isolated element approach, surrounded element approach, and unit cell analysis with PBC) can degrade due to the effect of the real mutual coupling. On the other hand, the reflectarray designed using the proposed method shows no performance degradation because the effect of real mutual coupling has already been dealt with exactly. 


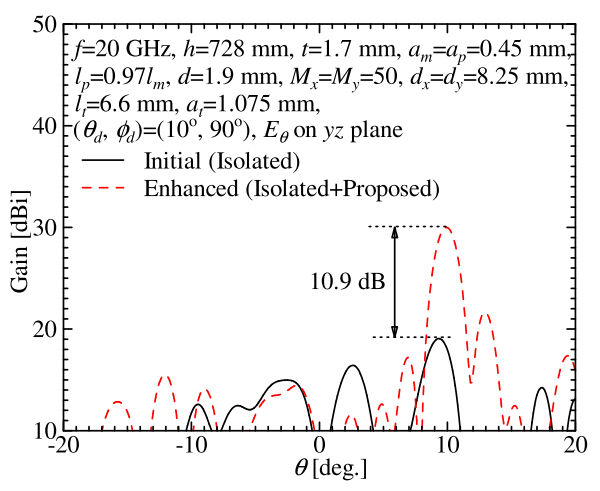

(a) Isolated, $d_{x}=d_{y}=0.55 \lambda$.

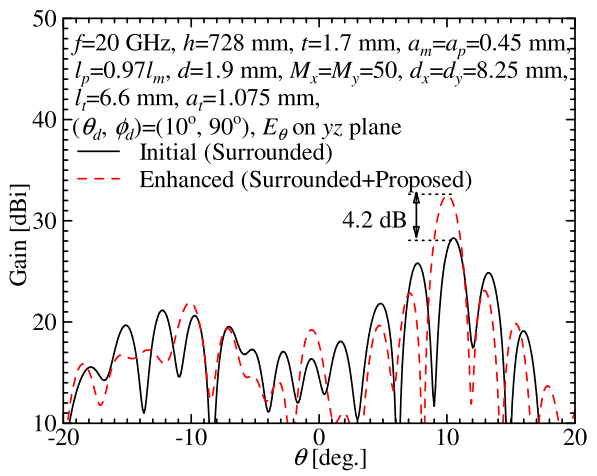

(c) Surrounded, $d_{x}=d_{y}=0.55 \lambda$.

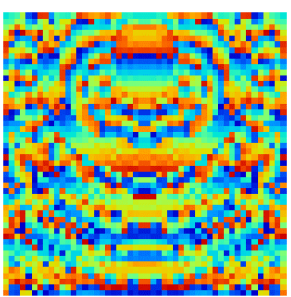

(a) Initial

$-180 \quad$ [deg.] 180

(e) Isolated, $d_{x}=d_{y}=0.55 \lambda$

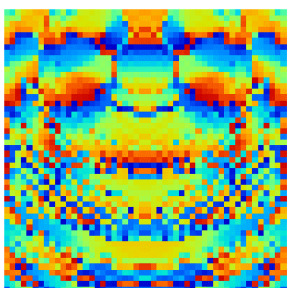

(a) Initial

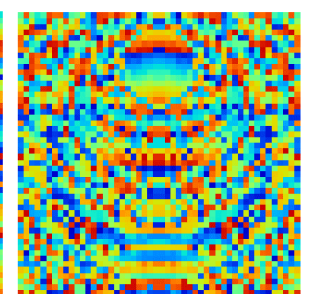
(b) Enhanced

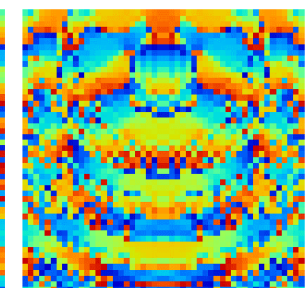

$-180 \quad$ [deg.] 180

(g) Surrounded, $d_{x}=d_{y}=0.55 \lambda$.

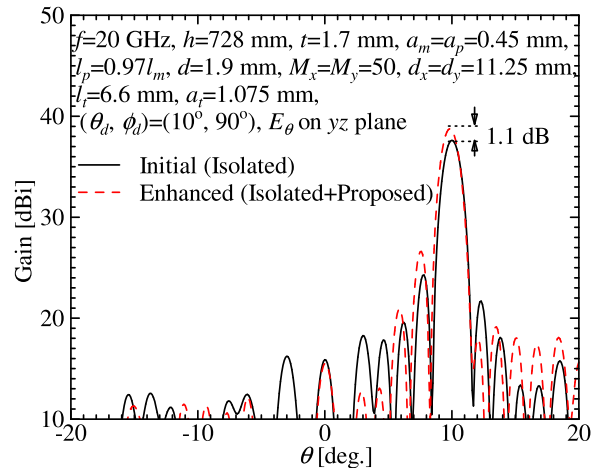

(b) Isolated, $d_{x}=d_{y}=0.75 \lambda$.

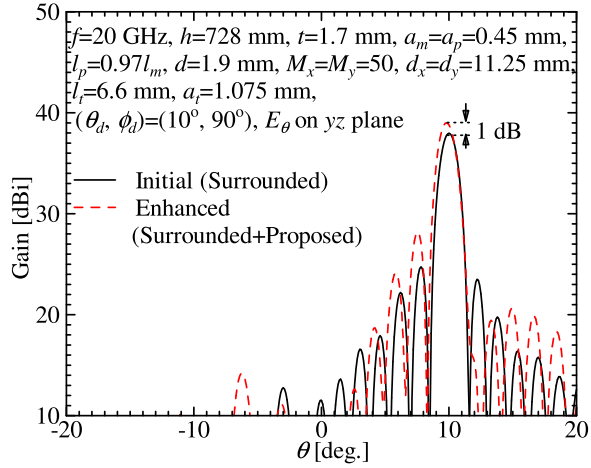

(d) Surrounded, $d_{x}=d_{y}=0.75 \lambda$.

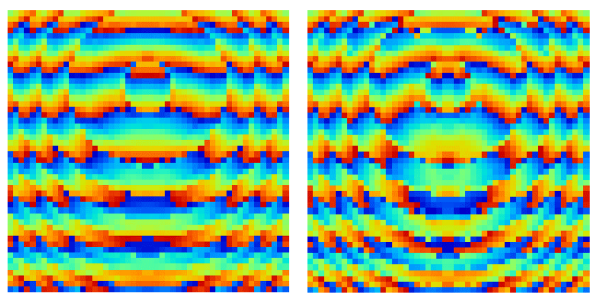

(a) Initial (b) Enhanced

(f) Isolated, $d_{x}=d_{y}=0.75 \lambda$.

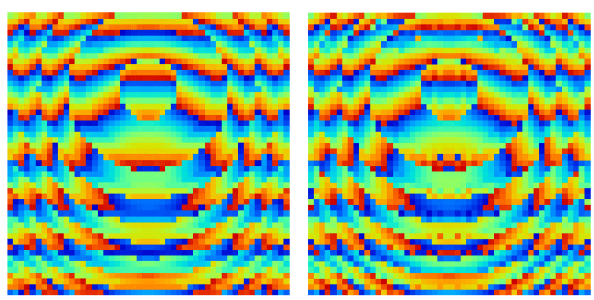

(a) Initial (b) Enhanced

Fig. 2. Gain and aperture phase distribution of $50 \times 50$ reflectarray designed by isolated/surrounded element approach $(F / D=1)$.

Summary of the design examples is shown in Table I. It is found that the gain and aperture efficiency of the reflectarrays designed using the surrounded element approach are higher than those designed using the isolated element approach when the array spacing is the same. Therefore, the reflectarrays designed using the

๑) IEICE 2016

DOI: 10.1587/comex.2016XBL0116 Received June 10, 2016 Accepted June 22, 2016 Publicized July 7, 2016 Copyedited September 1, 2016 
surrounded element approach are assumed to be better initial structures than those designed using the isolated approach. As a result, relatively high gain and high aperture efficiency are achieved using the proposed method at the expense of small computational cost when the initial reflectarrays are designed using the surrounded element approach, not the isolated element approach.

Table I. Summary of design examples $\left(F / D=1,\left(\theta_{d}, \phi_{d}\right)=\left(10^{\circ}, 90^{\circ}\right)\right.$, $50 \times 50$ array).

\begin{tabular}{l|l|l|l|l}
\hline Initial design of reflectarrays & \multicolumn{2}{|c|}{ Isolated } & \multicolumn{2}{c}{ Surrounded } \\
\hline Array spacing $d_{x}=d_{y}[\lambda]$ & 0.55 & 0.75 & 0.55 & 0.75 \\
\hline Gain [dBi] & 30 & 38.7 & 32.5 & 39 \\
(w/o proposed method) & $(19.1)$ & $(37.6)$ & $(28.3)$ & $(38)$ \\
\hline Aperture efficiency [\%] & 11 & 44.1 & 19.4 & 46.8 \\
(w/o proposed method) & $(0.9)$ & $(34)$ & $(7.4)$ & $(36.9)$ \\
\hline Total number of unknowns & 10,858 & 10,858 & 11,466 & 10,946 \\
\hline Total CPU time [sec.] & 105,508 & 36,374 & 52,156 & 37,935 \\
\hline Total number of full-wave simulations & 5,029 & 4,333 & 3,211 & 4,577 \\
\hline CPU time per full-wave simulation [sec.] & 21 & 8.4 & 16.2 & 8.3 \\
\hline
\end{tabular}

\section{Conclusion}

In this letter, the aperture efficiency of reflectarrays was enhanced using the efficient full-wave solver. It was shown that the accurate evaluation of real mutual coupling in reflectarray design enhance the aperture efficiency of the reflectarray at the expense of CPU time. In particular, the proposed method is quite helpful to enhance the gain of reflectarrays when their array spacing is small. The proposed method can be easily modified corresponding to various design targets because a full-wave solver and objective function in the proposed method can be replaced by the other one.

\section{Acknowledgments}

We would like to thank staffs in Cyberscience Center, Tohoku University for their helpful advices. This research used computational resources of the HPCI system provided by Cyberscience Center, Tohoku University through the HPCI System Research Project (Project ID:hp140052). This work was financially supported by JSPS KAKENHI Grant Number 26820137 and JSPS Postdoctral Fellowships for Research Abroad. 